\title{
Probabilistic Thermal Analysis During Mars Reconnaissance Orbiter Aerobraking
}

\author{
John A. Dec* \\ NASA Langley Research Center, Hampton, Virginia, 23681
}

\begin{abstract}
A method for performing a probabilistic thermal analysis during aerobraking has been developed. The analysis is performed on the Mars Reconnaissance Orbiter solar array during aerobraking. The methodology makes use of a response surface model derived from a more complex finite element thermal model of the solar array. The response surface is a quadratic equation which calculates the peak temperature for a given orbit drag pass at a specific location on the solar panel. Five different response surface equations are used, one of which predicts the overall maximum solar panel temperature, and the remaining four predict the temperatures of the solar panel thermal sensors. The variables used to define the response surface can be characterized as either environmental, material property, or modeling variables. Response surface variables are statistically varied in a Monte Carlo simulation. The Monte Carlo simulation produces mean temperatures and 3 sigma bounds as well as the probability of exceeding the designated flight allowable temperature for a given orbit. Response surface temperature predictions are compared with the Mars Reconnaissance Orbiter flight temperature data.
\end{abstract}

\section{Nomenclature}

$\begin{array}{ll}A L C_{p} & =\text { Aluminum honeycomb core specific heat, } \mathrm{J} / \mathrm{kg}-\mathrm{K} \\ A L k & =\text { Aluminum honeycomb core thermal conductivity, } \mathrm{W} / \mathrm{m}-\mathrm{K} \\ b_{i j} & =\text { Response surface equation coefficients } \\ b_{0} & =\text { Response surface intercept coefficient } \\ C_{H} & =\text { Heat transfer coefficient } \\ C R & =\text { Contact resistance, } \mathrm{W} / \mathrm{m}^{2}-\mathrm{K} \\ D P & =\text { Drag pass duration, sec } \\ D O E & =\text { Design of Experiments } \\ F S C_{p} & =\text { M55J composite facesheet specific heat, } \mathrm{J} / \mathrm{kg}-\mathrm{K} \\ F S E & =\text { M55J composite facesheet emissivity } \\ F S k & =\text { M55J composite facesheet thermal conductivity, } \mathrm{W} / \mathrm{m}-\mathrm{K} \\ I T J E & =\text { ITJ solar cell emissivity } \\ I T & =\text { Initial solar panel temperature, }{ }^{\circ} \mathrm{C} \\ M D & =\text { Solar cell mass distribution, } \mathrm{kg} \\ M G S & =\text { Mars Global Surveyor } \\ M O I & =\text { Mars orbit insertion } \\ M R O & =\text { Mars Reconniassance Orbiter } \\ O F M & =\text { Outboard panel M55J facesheet mass distribution, } \mathrm{kg} \\ Q_{s} & =\text { Solar and planetary heat flux, } \mathrm{W} / \mathrm{cm}^{2} \\ R^{2} a d j & =\text { R squared adjusted } \\ T_{m} & =\text { Temperature }\left({ }^{\circ} \mathrm{C}\right) \text { of the } \mathrm{m}^{\text {th }} \text { point on the solar array } \\ V & =\text { Periapsis velocity, } \mathrm{km} / \mathrm{s} \\ X_{i} & =\text { Independent variable } \\ X_{j} & =\text { Independent variable } \\ \Delta V & =\text { Change in velocity, } \mathrm{km} / \mathrm{s} \\ \rho_{\infty} & =\text { Freestream density, } \mathrm{kg} / \mathrm{km}^{3} \\ & \end{array}$

"Aerospace Engineer, Structural and Thermal Systems Branch, MS 431, AIAA Member. 


\section{Introduction}

$\mathrm{T}$

HERE are numerous challenges associated with placing a spacecraft in orbit around Mars. Often, trades must be made such as the mass of the payload and the amount of fuel that can be carried. One technique employed to more efficiently place a spacecraft in orbit while maximizing payload mass (minimizing fuel use) is aerobraking. Aerobraking is a technique by which a spacecraft makes successive passes into a planet's atmosphere. Atmospheric drag reduces the spacecraft's periapsis velocity thereby lowering the apoapsis altitude and velocity on each pass through the atmosphere. A larger drag, results in a larger $\Delta \mathrm{V}$ for a given pass. The spacecraft passes through the upper atmosphere at hypersonic speeds and as a result is subjected to an aerodynamic heat load. This aerodynamic heat load causes the temperature of the spacecraft and its components to increase during the drag pass. To make an obvious observation, the higher the incident heat flux, the higher the resulting temperature. The atmospheric drag and the aerodynamic heating are both functions of the atmospheric density and spacecraft velocity. This results in one of the fundamental trades in performing an aerobraking maneuver, that is, as the spacecraft passes deeper into the atmosphere, the density increases, which results in more drag and a larger $\Delta \mathrm{V}$. However, at the same time, as the density increases, so does the aerodynamic heating, which results in higher spacecraft temperatures. Most science orbiters are typically designed to minimize structural mass in order to maximize the science payload. The materials used in the construction of these spacecraft have finite temperature limits that cannot be exceeded without loss of structural integrity or functional performance. The thermal limits then introduce a constraint to the aerobraking process which dictates how large the aerodynamic heating is allowed to become and thus how much $\Delta \mathrm{V}$ can be obtained on a given drag pass.

Aerobraking was first demonstrated by the Magellan spacecraft in orbit around Venus ${ }^{1}$. Mars Global Surveyor and Mars Odyssey both successfully performed aerobraking maneuvers around Mars ${ }^{2,3}$. The Mars Reconnaissance Orbiter also made use of aerobraking to gradually reduce its 45 hour post MOI orbit to its 2 hour science orbit. One thing all of these missions had in common was that during the drag pass, the solar array was the most limiting component in terms of its thermal limit, and it was this limit along with other constraints such as local mean solar time, etc. that determined how long it would take to reach the final desired orbit. Predicting the temperature of a specific spacecraft component during aerobraking is a challenging endeavor. There are many variables that need to be considered in such an analysis. The most obvious are the environmental heat loads on the component, and in particular, for MRO, the heat loads on the solar array. There are other variables that influence the analysis, but are less visible since they are associated with the thermophysical properties of the materials used in constructing the solar array. Finally, there are variables associated with the creation of a computer model as a representation of the physical object. All of these variables, the environment, material properties, and modeling variables have some uncertainty associated with them. The most notable, and arguably the most influential, in the thermal analysis during aerobraking, is the uncertainty in predicting the atmospheric density. Uncertainties in predicting the density can lead to large differences between the predicted temperature and the actual flight temperature encountered. Figure 1 shows the predicted density profile plotted with the actual density profile for an orbit pass encountered during Mars Odyssey aerobraking. Figure 1 shows that the actual density encountered is significantly different from the assumed density profile, and since heat flux and heat load are directly proportional to the density, there is therefore a difference in the peak heat flux reached and the total heat load, both of which affect the temperature. This type of variation has been observed in all of the Mars aerobraking missions. A probabilistic thermal analysis 


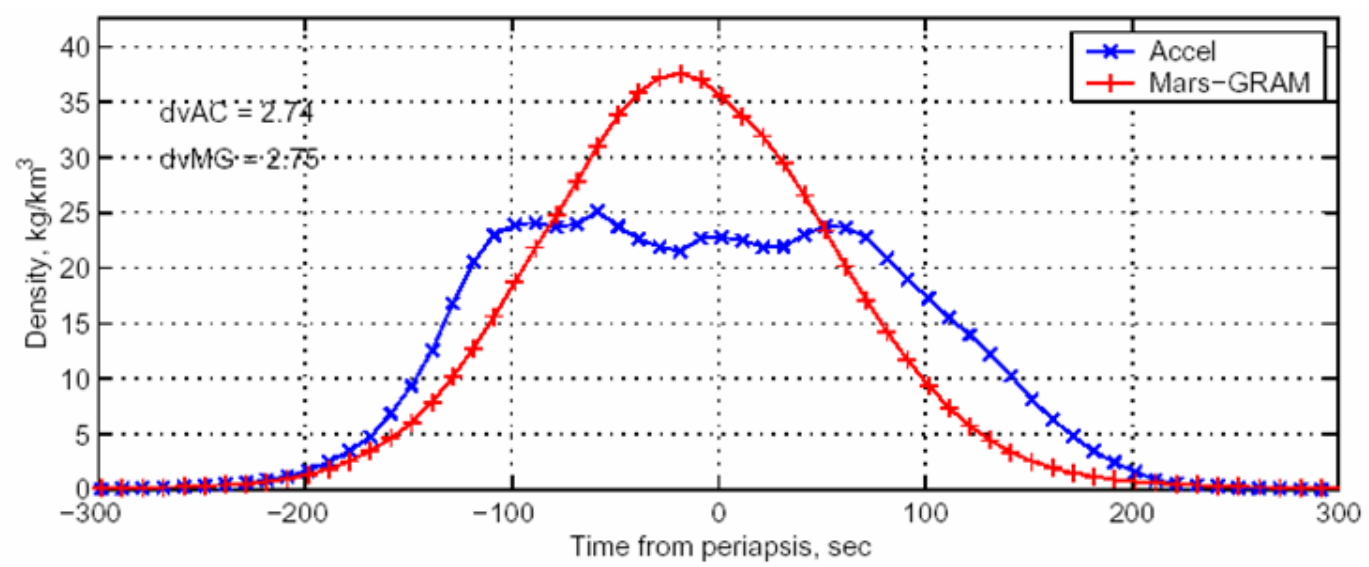

Figure 1. Mars Odyssey periapsis 157 density profile.

could incorporate the uncertainties in the most influential analysis variables and greatly increase the predictive power of the thermal analysis. However, a probabilistic analysis implies that a Monte Carlo simulation be performed. A Monte Carlo simulation is one in which the same calculation is performed repeatedly, but each time the input parameters to the calculation are statistically varied according to a specified distribution. Using a high fidelity finite element model to perform a Monte Carlo simulation is not practical. For example, for the MRO thermal model, a typical run analyzing the solar panel during a given drag pass takes approximately 1 hour. To perform a 10,000 case Monte Carlo simulation, which is considered a low number of samples, would take 10,000 hours, or just over a year. A response surface model is a possible solution. A response surface is a simple quadratic equation that can be evaluated in seconds instead of hours. This kind of fast calculation suits a Monte Carlo simulation. The ability to calculate the temperatures rapidly also facilitates onboard spacecraft calculations of the temperature, which could in turn be used in autonomous aerobraking. ${ }^{4}$

\section{Approach}

The general approach uses the high fidelity finite element thermal model of the MRO solar array which was used during its design phase to estimate its thermal limit. Based on the high fidelity model, a meta-model can be constructed using the response surface technique. A response surface is simply an equation derived by performing a regression of a set of data. The data in this case comes from performing several runs of the PATRAN Thermal model of the solar array. A design of experiments (DOE) is performed which determines the number of runs that must be performed and at what values the variables of interest should be set. Once the response surface equations have been derived, they are used in a Monte-Carlo simulation which statistically varies the analysis variables and calculates a mean and 3 sigma temperature. The Monte Carlo simulation also keeps track of all the samples that cause the temperature to exceed the flight allowable temperature limit and calculates the probability of an over temperature condition.

\section{A. Thermal Model Background}

The MRO thermal model is based off of the Patran and Thermal Desktop thermal models developed for the Mars Global Surveyor and Mars Odyssey spacecraft, ${ }^{5,6}$. A companion paper describes the finite element thermal model development for MRO so the details will not be discussed in this paper ${ }^{7}$. The thermal model for Mars Global Surveyor was developed after the spacecraft had already successfully performed its aerobraking maneuver around Mars and roughly 2 months prior to the development of the Mars Odyssey thermal model. Since the Mars Global Surveyor model could be correlated to flight data, it provided an excellent means of validating the model and methods to be used for Odyssey. The goal of the Mars Odyssey model prior to aerobraking operations was verify and establish thermal limits for the solar array. The thermal limits define the maximum heat flux the solar arrays can encounter and not exceed their flight allowable temperature. In addition, the Odyssey model was used during the aerobraking phase of the mission to predict solar array temperatures for each drag pass. The MRO thermal model was developed and used similarly to the Mars Odyssey model and was made up of the same three basic components. The first was a Thermal Desktop model which was used to calculate the radiation view factors and the orbital heating during each orbit. The second was a Patran Thermal finite element model which was used to calculate 
temperatures during the drag pass. Finally, a Direct Simulation Monte Carlo (DSMC) model of the spacecraft and solar arrays was used to calculate the heat transfer coefficients as a function of atmospheric density. The Thermal Desktop and DSMC output as well as a given POST flight trajectory make up the boundary conditions used by the Patran thermal model to calculate temperatures. Figure 2 shows the Thermal Desktop and Patran thermal models on the left and right respectively. The MRO thermal models are detailed and attempt to capture all of the significant
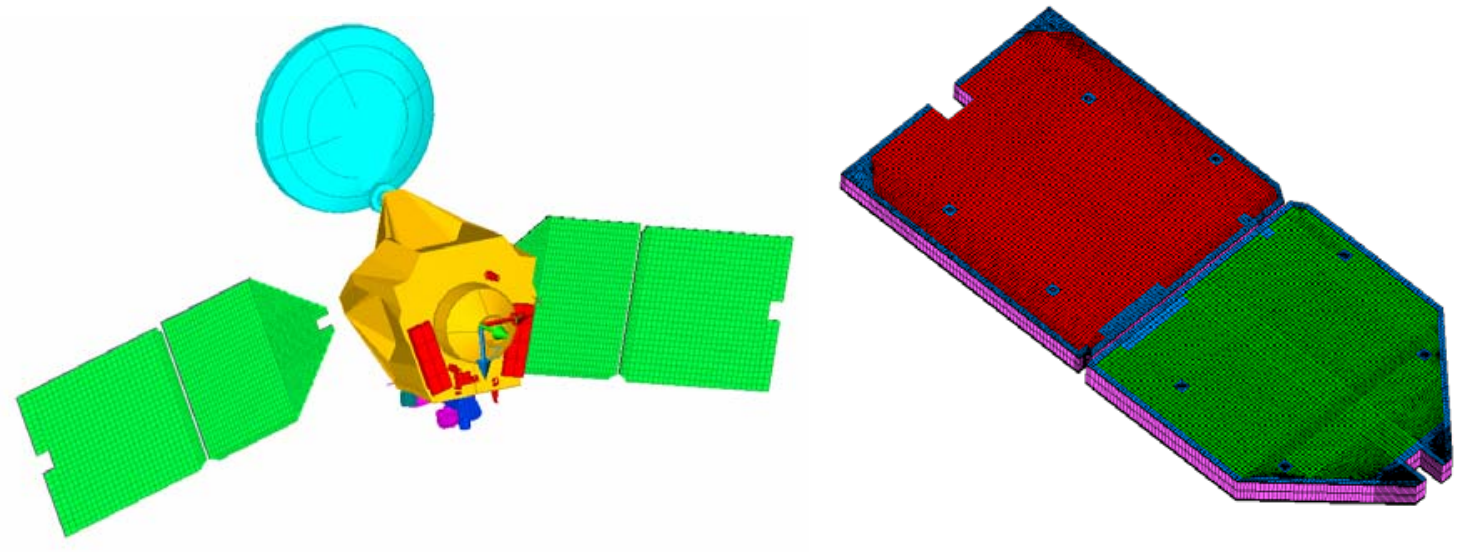

Figure 2. Thermal Desktop (left) and Patran Thermal model (right)

design features of the spacecraft and solar arrays. The down side of modeling small details is in the run time required to obtain a solution for the temperatures. The Patran Thermal model running on a $2.6 \mathrm{Ghz}$ Xeon processor requires on average approximately 1 hour per drag pass. The Thermal Desktop simulations are slightly more efficient running on the same system as above and require about 30 minutes for one orbit. Figure 3 shows the heat transfer coefficient $\left(\mathrm{C}_{\mathrm{H}}\right)$ distribution for a density of $32 \mathrm{~kg} / \mathrm{km}^{3}$. Figure 3 also shows the locations of the solar array

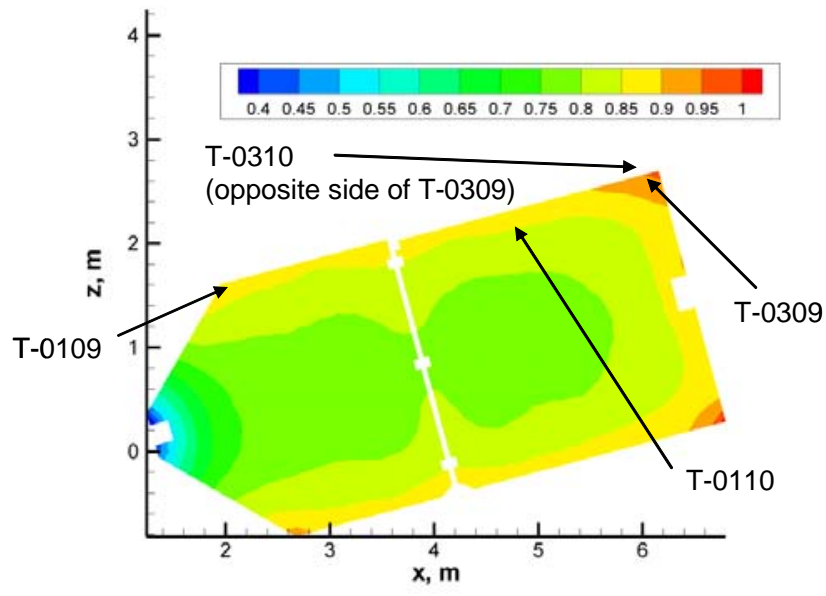

Figure 3. MRO heat transfer coefficient distribution with sensor locations for a density of $32 \mathrm{~kg} / \mathrm{km}^{3}$

temperature sensors and indicates that they are located in the regions which are anticipated to exhibit the highest temperatures during flight. Putting all the modeling elements together, the temperatures of the solar array can be calculated. Figure 4 shows the predicted temperature distribution calculated for orbit pass 64 and confirms that the temperature sensors are located in regions where the temperature is the highest. The utility of the thermal response surface analysis requires that a high fidelity thermal model be developed in order to ensure its accuracy and so that it may capture as many of the uncertainties in the analysis as possible. In other words it would be difficult to construct accurate thermal response surface equations without first developing the high fidelity thermal models. 

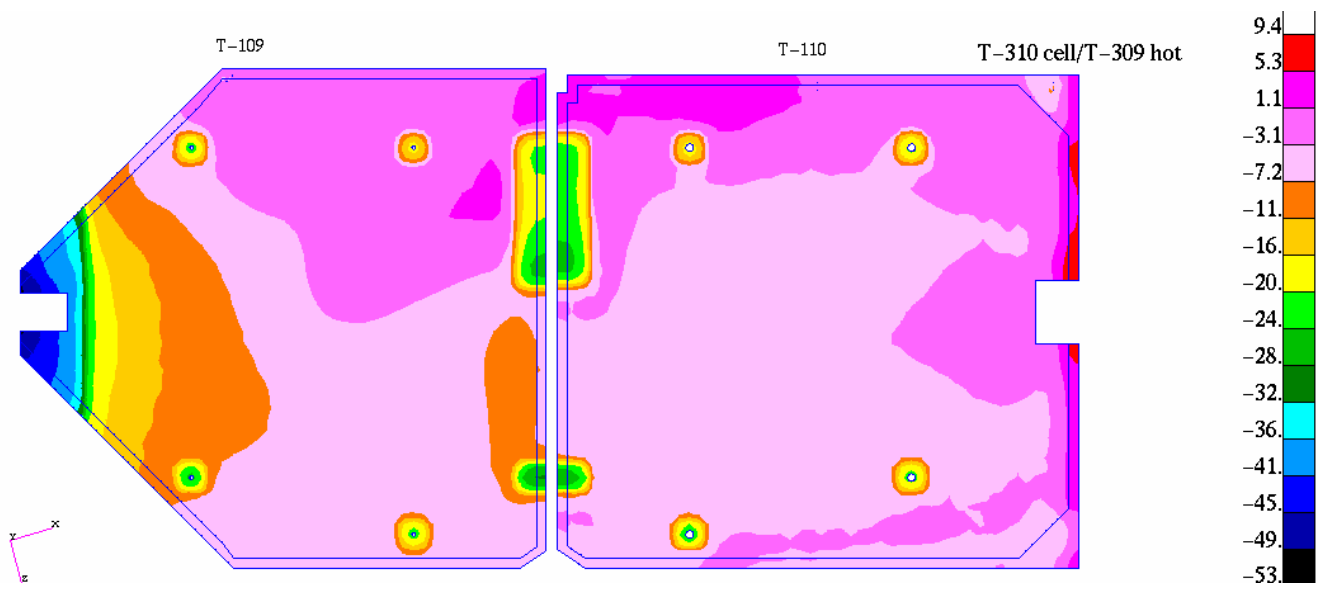

Figure 4. Predicted MRO temperature distribution $\left({ }^{\circ} \mathrm{C}\right)$ for orbit pass 64

The Odyssey thermal model was also correlated to flight data and much insight was gained into how to construct thermal models for aerobraking operations. ${ }^{8}$ The Odyssey model correlation to flight data also reveled that the drag pass temperature predictions were directly linked to how accurate the atmospheric density predictions were for each drag pass. Large uncertainties in the density predictions caused the predicted temperatures to deviate significantly from the flight temperature data for a given drag pass. Referring back to Figure 1, the difference between the predicted density and the actual density is clearly shown. This difference exits for all orbit passes but varies in magnitude from orbit to orbit. Given the uncertainty in predicting the density, there was a low confidence in the predicted temperatures, and as such they were not very useful for maneuver planning. Although the density had the greatest affect on the temperature, other input parameters associated with the environment, thermophysical properties and modeling correlations (e.g. contact resistance) also contributed to the difference between the flight temperatures and the predictions. In order to improve the temperature predictions for MRO and make them more useful to the flight navigation team, a new method for calculating the temperatures during a drag pass was needed. A probabilistic analysis in which a Monte Carlo simulation is used to statistically vary the input variables is such a method.

\section{B. Response Surface Development}

As mentioned previously, the run times for the Patran thermal model make using it in a Monte Carlo simulation impractical. In order to run a Monte Carlo simulation, the temperatures need to be calculated in seconds rather than hours. A solution to this problem and in fact an enabler to this analysis method is a response surface model. A response surface model is usually a linear or quadratic equation that can be used to determine how a given response is affected by a set of quantitative independent variables or factors over some specified range. Equation 1 shows the general form for a quadratic response surface equation with terms for two and three-variable interactions. A response surface equation can take any mathematical form, but there are clear advantages to using a polynomial. One advantage of using a polynomial response surface equation is that it can be evaluated several times per second. Another advantage is that taking derivatives of a polynomial is trivial which facilitates sensitivity analysis.

$$
T_{m}=b_{0}+\sum_{i=1}^{n} b_{i} x_{i}+\sum_{i=1}^{n} b_{i i} x_{i}^{2}+\sum_{i=1}^{n-1} \sum_{j=i+1}^{n} b_{i j} x_{i} x_{j}+\sum_{i=1}^{n-2} \sum_{j=i+1}^{n-1} \sum_{k=j+1}^{n} b_{i j k} x_{i} x_{j} x_{k}
$$

In the case of the thermal analysis of the MRO solar arrays during aerobraking, the response surface equations are quadratic with only two-variable interaction terms present, the reasoning for this will become clear later. The dependant variable of the equation is the solar array temperature at a given location on the array. To analyze multiple points on the array, multiple response surface equations are required.

Once the form of the response surface equation has been established, the independent variables used in the equation need to determined. Determining what variables to use and the number of variables to consider in 
constructing the response surface is a trade that must be performed. Determining which variables to use is straight forward and is typically done by having a brainstorming session with discipline experts. While this method is the least time consuming, it is the most flawed in that some variables might have a large influence and not get selected to be in the analysis. A more rigorous method would be to perform a sensitivity analysis for each variable and each interaction with the other variables. ${ }^{9}$ This sensitivity analysis would also reveal if any correlation exists between the variable, which in this analysis they are assumed to be uncorrelated and can vary independently. Performing the sensitivity analysis would increase the fidelity of the analysis, but would also be time consuming. In the case of MRO, due to time constraints, the brainstorming method was used where the analysts involved in developing, using, and correlating the thermal models for MGS and Mars Odyssey determined which of the input variables to the MRO thermal analysis had the most influence on the calculated temperature and had the most uncertainty. Ideally, one would like to select all the variables that exhibit any kind of influence on the temperature, however, the number of variables used in response surface equation directly determines the number of Patran Thermal runs that must be performed. The 15 independent variables, or factors selected for the MRO response surface are listed in Table 1 along with their $3 \sigma$ uncertainty. The upper and lower bounds for the variables used to define the response surface were $\pm 6 \sigma$ except for cases where a $6 \sigma$ range would give the variable a value that was not realistic. The M55J and ITJ the emissivities are examples of this, where a $+6 \sigma$ variation would cause the emissivity to be greater than 1.0

Table 1. MRO analysis variable used in developing the response surface equation

\begin{tabular}{clccc}
\hline \hline \multirow{2}{*}{ Category } & \multicolumn{1}{c}{ Factor } & $\begin{array}{c}3 \sigma \\
\text { uncertainty }\end{array}$ & $\begin{array}{c}\text { Response } \\
\text { Surface } \\
\text { Bounds }\end{array}$ & Distribution \\
& & $\pm 3 \%$ & $\pm 6 \sigma$ & Normal \\
Environmental & Drag pass duration & $\pm 30 \%$ & $\pm 6 \sigma$ & Gamma \\
& Density & $\pm 14 \%$ & $\pm 6 \sigma$ & Uniform \\
& Heat transfer coefficient & $\pm 0.05 \mathrm{~km} / \mathrm{s}$ & $\pm 6 \sigma$ & Normal \\
& Periapsis velocity & $\pm 20.0{ }^{\circ} \mathrm{C}$ & $\pm 6 \sigma$ & Normal \\
& Initial solar array temperature & $\pm 30 \%$ & $\pm 6 \sigma$ & Normal \\
& Orbital heat flux & $\pm 8 \%$ & $\pm 5 \sigma$ & Normal \\
Material & M55J graphite emissivity & $\pm 10 \%$ & $\pm 5 \sigma$ & Normal \\
& Property solar cell emissivity & $\pm 25 \%$ & $\pm 6 \sigma$ & Normal \\
& M55J graphite thermal conductivity & $\pm 15 \%$ & $\pm 6 \sigma$ & Normal \\
& M55J graphite specific heat & $\pm 30 \%$ & $\pm 6 \sigma$ & Normal \\
& Aluminum honeycomb core thermal conductivity & $\pm 5 \%$ & $\pm 6 \sigma$ & Normal \\
& Aluminum honeycomb core specific heat & $\pm 50 \%$ & $\pm 5 \sigma$ & Normal \\
Modeling & Outboard solar panel mass distribution & $\pm 10 \%$ & $\pm 5 \sigma$ & Normal \\
& Solar cell layer mass distribution & $\pm 50 \%$ & $\pm 6 \sigma$ & Normal \\
\hline \hline
\end{tabular}

which is not possible. The best way to determine the minimum required number of Patran thermal model runs is to perform a statistical design of experiments (DOE). There are many available to choose from, the most complete being the full factorial design in which each variable value combination is run. A full factorial design represents the upper bound on the number of Patran runs needed to perform a regression and hence generate a response surface equation. The number of runs for a full factorial is related to the number of variables being considered as well as how many discrete values, (or levels), the variable will take in the analysis. For example if we were considering 4 variables and wished to use two levels, (i.e. set them at their high and low value), the number of runs required for a full factorial would be $2^{4}$, or 16 , for 5 variables using three levels (high, low, and midpoint value), it would be $3^{5}$, or 243 runs. From these small examples it is clear that as the number of variables and their discrete values increases the number of required runs increases exponentially. This is problematic if there are a lot of variables that need to be considered, as in the case of the MRO thermal model. In addition, if multiple interaction terms are present, more runs would be required. For 15 variables at three levels the number of runs would be 14,348,907, a very impractical amount. This is also why it is not practical to select every variable that exhibits influence on the temperature, only the most influential should be selected. Fortunately, the statistical software JMP has the ability to create other DOE's that minimize the thermal model runs needed while maintaining the regression model's accuracy. JMP was used to perform a statistical design of experiments (DOE) using a face centered central composite design to determine the number of runs and what values those variables would take for each run. One of the constraints in 
place that needed to be dealt with was time. It was desired that all of the required Patran runs be complete in two days, that means that at most, using a 10 node computer cluster, 480 runs could be completed. This constraint was in place because if the thermal model was changed, the response surface equations would have to be updated to reflect that change. A face centered central composite DOE provided the necessary accuracy accommodating all of the selected variables using three levels while minimizing the number of runs required, which turned out to be 296 runs. After performing the 296 Patran runs, where for each run the 15 variables were set to different values, the peak temperature results for the four thermal sensors and the overall maximum temperature were input into JMP. Using JMP's step-wise regression functionality, the coefficients to the response surface equations were determined, one equation for each temperature of interest. The temperature results for the maximum temperature obtained by using the response surface equation compare very well with the results obtained from the Patran run. Figure 5 shows the error distribution between the response surface and the Patran model for the 296 runs. Figure 5 also shows that $95.6 \%$ of the results are within $\pm 10^{\circ} \mathrm{C}$, which is a very acceptable result. The overall root mean square error for the response surface equation for the maximum

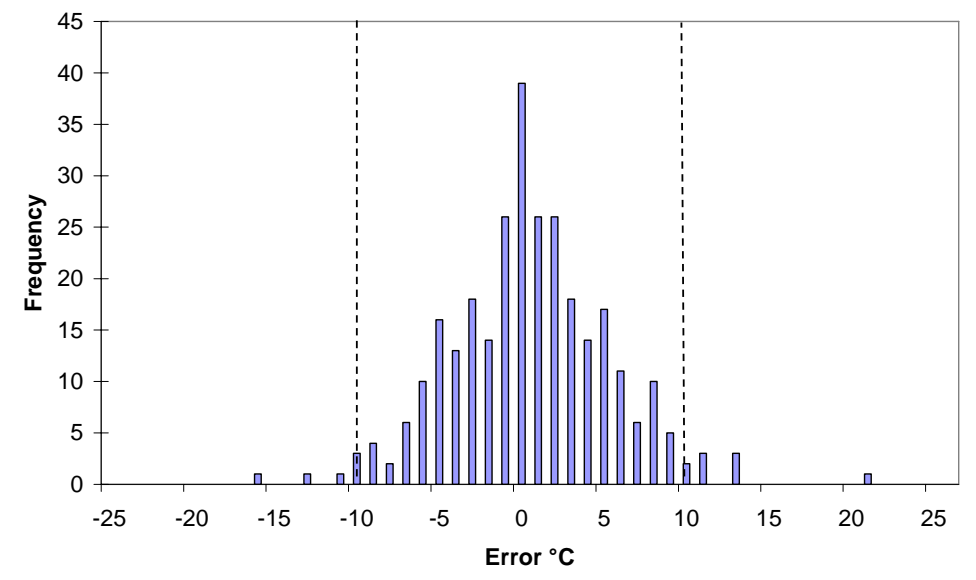

Figure 5. Error distribution between response surface and Patran model maximum temperature

temperature is $3.4^{\circ} \mathrm{C}$ with and $\mathrm{R}^{2}$ adjusted of 0.997 indicating a very good fit exists between the Patran model and response surface equation. Similar RMS errors and $\mathrm{R}^{2}$ adjusted are obtained for the 4 thermal sensors as well and are summarized in Table 2.A more rigorous analysis of the fit and error, as well as determining the statistical significance of each variables contribution to the temperature response can be performed using JMP. A discussion of the aforementioned analysis is beyond the scope of this current work.

Table 2. RMS error and $\mathbf{R}^{2}$ adjusted for response surface equation fit of the Patran thermal model

\begin{tabular}{lcc}
\hline Temperature/Sensor & RMS Error ${ }^{\circ} \mathrm{C}$ & $\mathrm{R}^{2}$ Adj. \\
\hline $\mathrm{T}_{\max }$ & 3.44 & 0.997 \\
$\mathrm{~T}-0109$ & 2.07 & 0.998 \\
$\mathrm{~T}-0110$ & 2.37 & 0.997 \\
$\mathrm{~T}-0309$ & 2.06 & 0.998 \\
$\mathrm{~T}-0310$ & 2.67 & 0.990 \\
\hline \hline
\end{tabular}

The last step is to determine the uncertainties and perform a Monte Carlo simulation. Estimating the uncertainty is probably the most difficult and the most critical part in conducting a probabilistic analysis. Improper or nonconservative estimates of the uncertainties can lead to erroneous results. Wright, et al. ${ }^{9}$ identified six different methods for quantifying uncertainty, 2 of which have been used to estimate the uncertainty for the variables of this analysis. For the drag pass duration, atmospheric density, heat transfer coefficient and periapsis velocity, the uncertainties were determined by analyzing past Mars aerobraking mission data and computing the mean and standard error of the data for each. This is by far the most rigorous and accurate method of quantifying the uncertainty. For all of the material property variables, as well as the modeling variables, the initial temperature, and 
orbital heating, the uncertainties were estimated by expert judgment. Expert judgment is the least rigorous and most prone to error which could give low confidence in the results of the Monte-Carlo simulation. When applying this method it is best to err on the conservative side, which was the approach taken for this analysis. Referring back to Table 1, the estimated uncertainties for each variable are shown. Fortunately, the most influential variables which are the density, $\mathrm{C}_{\mathrm{H}}$, and the interaction between density and $\mathrm{C}_{\mathrm{H}}$ account for about $62 \%$ of the variation in the temperature response. Since the uncertainty for those variables was rigorously defined, there is a higher confidence in the resulting Monte-Carlo analysis.

\section{Results}

With the polynomial response surface equations it is straight forward to incorporate them into a Monte-Carlo simulation. The inputs to the simulation are the same as would be used to calculate the temperature via the response surface equation. The results of the Monte-Carlo simulation are the mean temperatures for the four thermal sensors and the maximum solar array temperature. The simulation also provides the standard deviation for the temperature, from which $\pm 3 \sigma$ temperature bounds can be calculated. Moreover, by keeping track of how many samples in the Monte-Carlo simulation cause the solar array temperature to exceed the flight allowable temperature of $175^{\circ} \mathrm{C}$, the probability of exceeding it can be estimated. During aerobraking operations, the only variables that change from orbit to orbit are the drag pass duration, density, periapsis velocity, and initial solar array temperature. The drag pass duration, density and periapsis velocity are generated directly from the flight mechanics simulations being performed for each orbit. The initial solar array temperature is determined from a correlation of orbital period versus temperature, where the orbital period is determined by the flight mechanics simulation. All of the other variables do not vary orbit to orbit and are set to their nominal values for the entire mission and vary according to their assumed distribution and uncertainty during the simulation. The results for the mean temperature predictions during aerobraking show that they match the flight data very well, and that the flight data falls within the calculated $3 \sigma$ temperature bounds. Figure 6 and 7 show the flight temperature for the "hottest" sensors plotted with the mean and $3 \sigma$ temperature prediction from the Monte Carlo analysis of the corresponding sensors. Figure 6 and 7 show that only a few data points fall outside the $3 \sigma$. They also show that even the few points that fall outside the bounds, they are not out by very much at all. Comparison of the other two sensors with flight data shows similar results which are show in Figures 8 and 9.

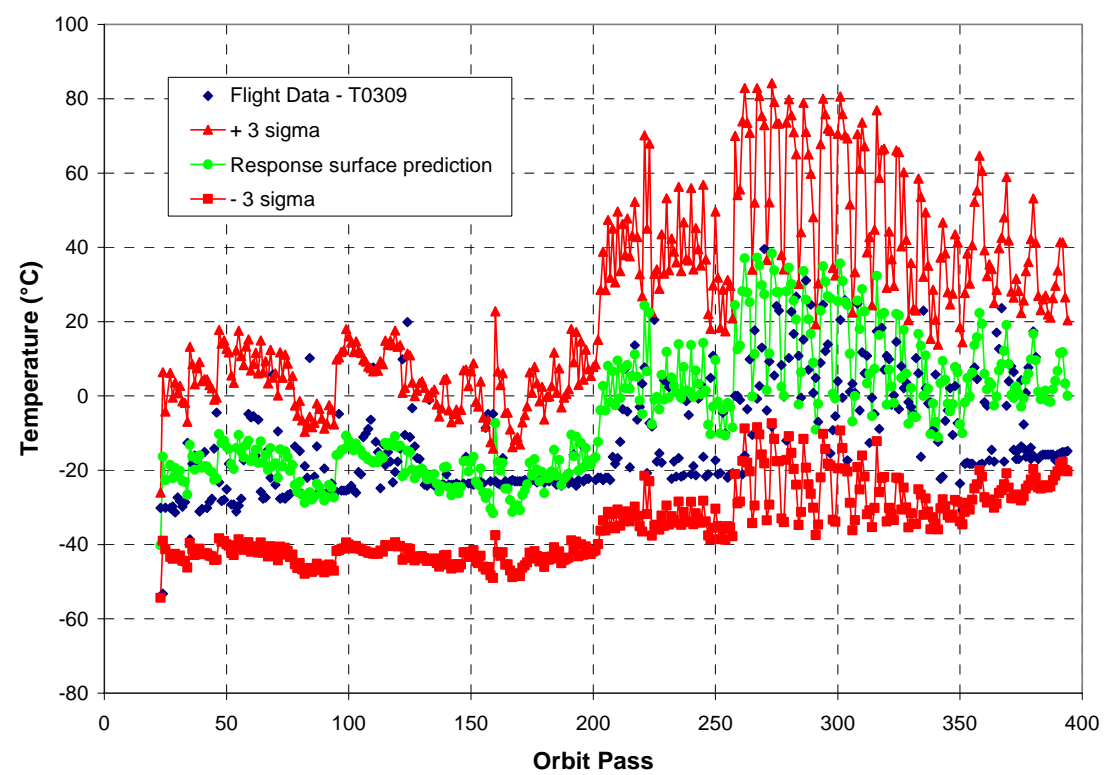

Figure 6. Comparison of response surface prediction with flight for sensor T-0309 


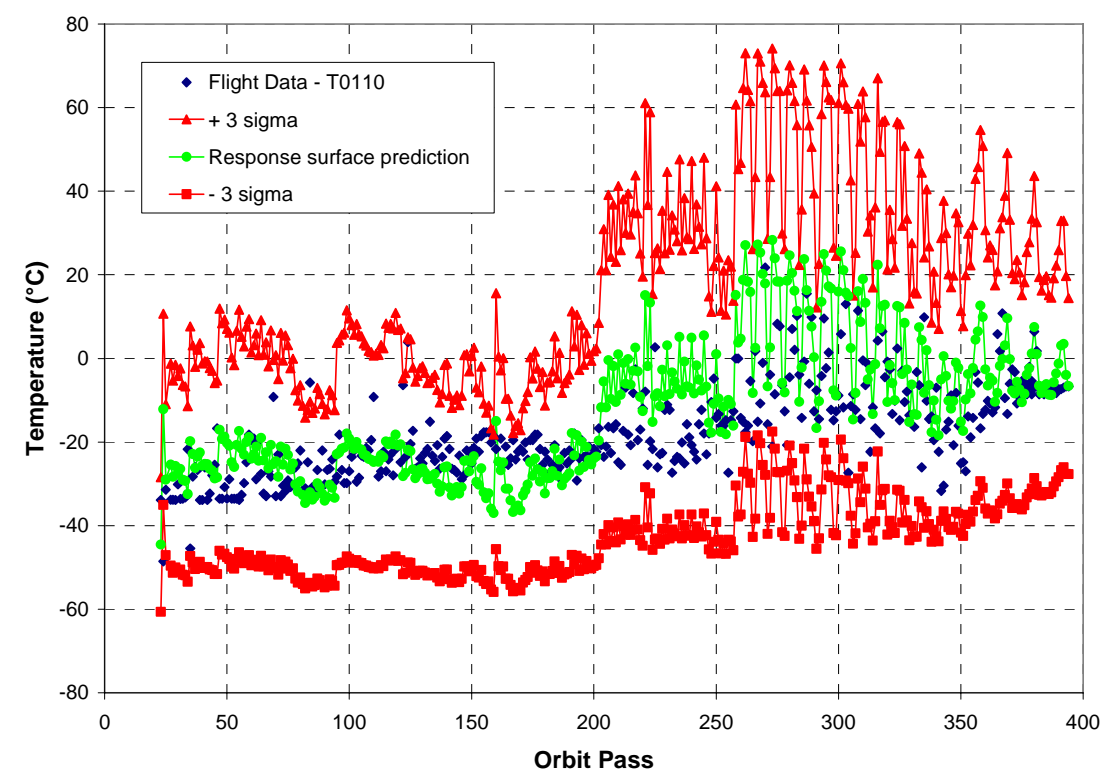

Figure 7. Comparison of response surface prediction with flight for sensor T-0110

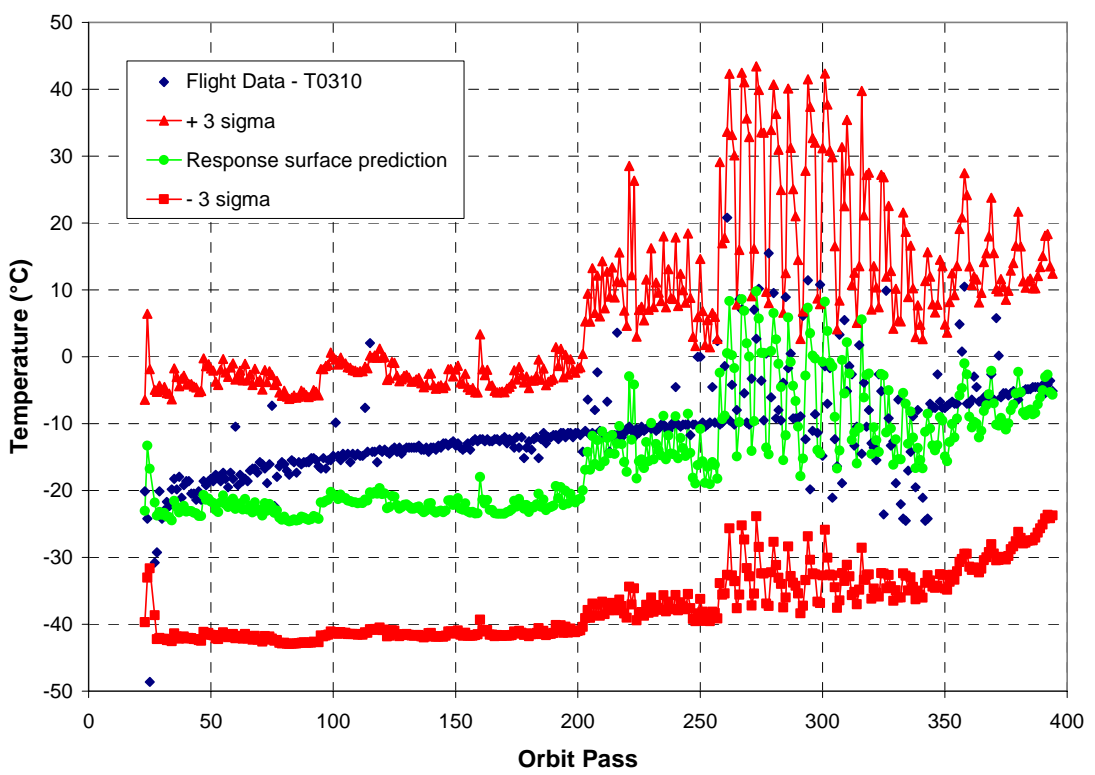

Figure 8. Comparison of response surface prediction with flight for sensor T-0310

Looking at the results it is clear that the mean and $3 \sigma$ temperature can both accurately predict and bound the temperature for any given drag pass. As good as these results are they are not perfect, the few cases where the flight temperature exceeded the $3 \sigma$ are examples of orbits that had large, sudden increases in density magnitude (i.e density spikes), or significant deviations away from the assumed Mars-GRAM density profile. These conditions are not modeled in either the Patran thermal model or the response surface equations and such conditions produce results that deviate significantly from the flight data. Another disadvantage of the response surface is that extrapolation outside the upper and lower bounds of any variable used in the response surface causes significant errors in the temperature calculation so extreme care must be take when supplying inputs to the response surface. Another example of how the response surface Monte-Carlo simulation can aid the maneuver decision making process, and an illustration of the response surface extrapolating can be shown in a mission run-out plot of the temperature over the remaining orbit passes. Figure 10 shows the temperature and the $3 \sigma$ upper bound from orbit 191 to 500. Mission planners used this data to assess the long term effect of performing maneuvers at specified orbits. Figure 10 also shows that towards the end of the mission, the drag pass durations start to exceed that defined 
by the upper bound of the response surface and show that the temperature erroneously starts to grow. Fortunately, the solar arrays ceased to be the most limiting component at around orbit pass 352 and as such the thermal analysis was no longer needed. In fact the final trajectory update from flight mechanics was for orbit 352 and included predictions of drag pass duration, density, velocity, and period up through orbit pass 392. Even without an updated trajectory, the response surface and Monte-Carlo simulation were able to predict the temperature fairly accurately. This has implications for autonomous aerobraking in the event that the spacecraft loses its ability to update the trajectory, the response surface can still be used determine the temperatures for a few orbit passes giving the flight operations team time to correct the anomaly.

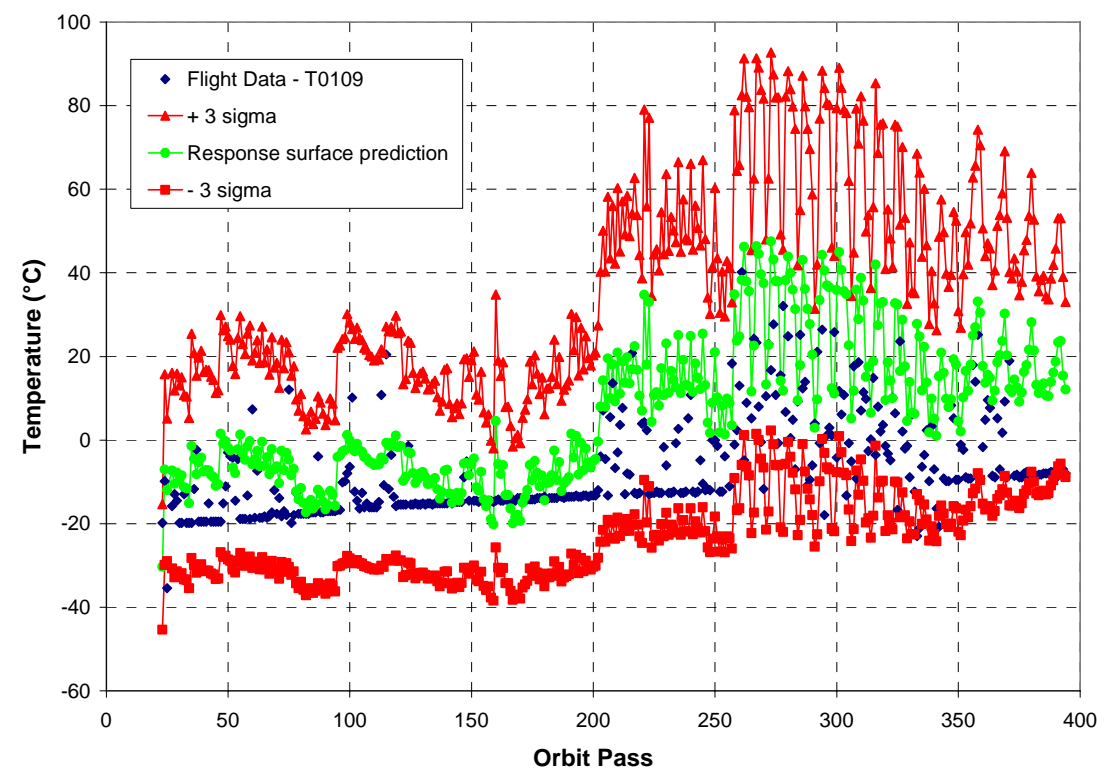

Figure 9. Comparison of response surface prediction with flight for sensor T-0109

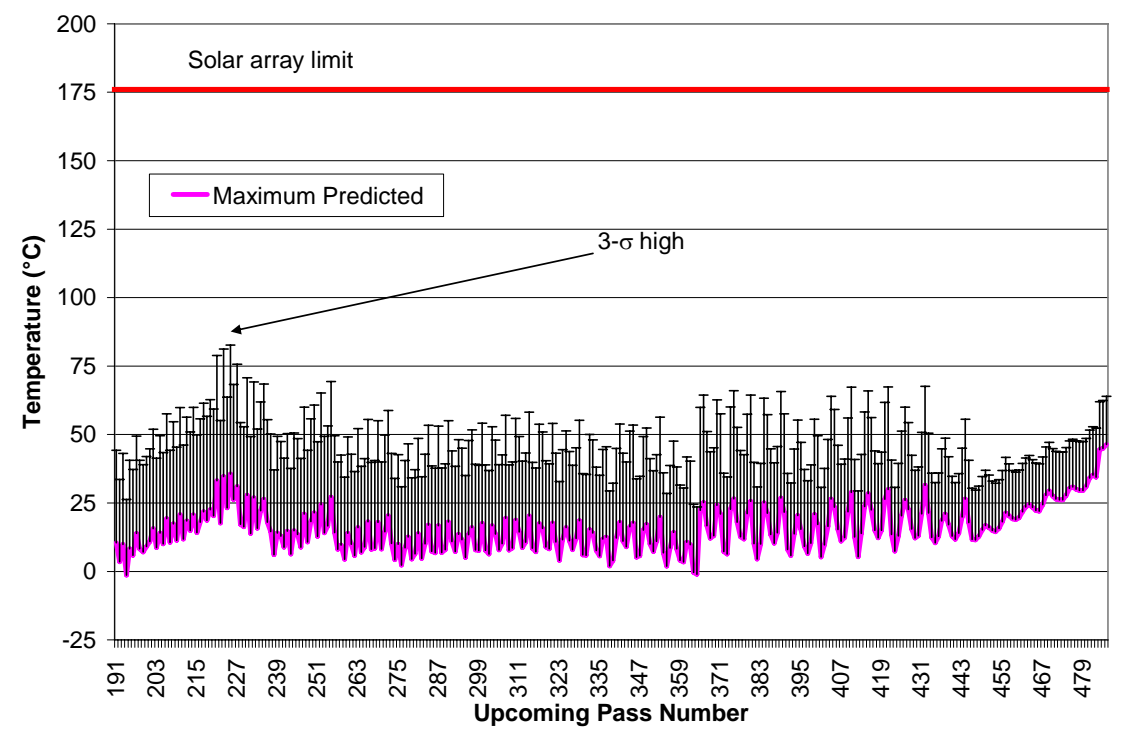

Figure 10. Mission run-out starting at orbit pass 191 


\section{Conclusion}

A methodology for developing a response surface model from a high fidelity thermal model was presented. Using the response surface equations, a Monte-Carlo simulation for probabilistically predicting the temperatures during aerobraking was developed. The primary objective was to improve the thermal analysis being performed during aerobraking operations and make the temperature predictions more useful for maneuver planning. Results show that the Monte-Carlo simulation accurately predicts and accurately bounds the temperature during an orbital drag pass during aerobraking thus increasing the utility of the thermal analysis. Furthermore, the response surface can be used to make temperature predictions for mission run-out scenarios, something that the high fidelity thermal can not do in a timely manner. Further research must be performed to allow modeling of orbit anomalies like density spikes, or density profiles which do not conform to the Mars-Gram profile. Methods for estimating the uncertainties must also be improved for this methodology to be adapted to autonomous aerobraking. More resources should be spent on making material property measurements, in order to 1) be able to rigorously define their uncertainties, and 2) to reduce the uncertainties that arise by using conservation expert judgment estimates.

\section{Acknowledgements}

The author would like to thank Scott Streipe for his support in allowing the MRO thermal team to develop this thermal analysis methodology. The author would also like to thank Dr. Alan Wihite, Dr. Robert Braun, of the Georgia Institute of Technology, and Dr. John Olds of Spaceworks Engineering, Inc. for their review of this work prior to aerobraking operations.

\section{References}

${ }^{1}$ Carpenter, A. S., "The Magellan Aerobraking Experiment: Attitude Control Simulation and Preliminary Flight Results”, AIAA Paper 93-3830, August 1993.

${ }^{2}$ Beerer, J., et. al., “Aerobraking at Mars: The MGS Mission”, AIAA $34^{\text {th }}$ Aerospace Sciences Meeting and Exhibit, Reno, Nevada, AIAA 96-0334, January 15-18, 1996.

${ }^{3}$ Smith, J. C., and Bell, J. L., “2001 Mars Odyssey Aerobraking”, Journal of Spacecraft and Rockets, Vol. 42, No. 3, 2005, pp. 406-415.

${ }^{4}$ Prince, J. L., Dec, J. A., and Tolson, R. H., "Autonomous Aerobraking Using Thermal Response Surface Analysis", AIAA-2007-861, AIAA 45 ${ }^{\text {th }}$ Aerospace Sciences Meeting and Exhibit, Reno, NV, January 8-11, 2007

${ }^{5}$ Amundsen, Ruth M., Dec, John A., and George, Benjamin, E. LT., "Aeroheating Thermal Model Correlation for Mars Global Surveyor (MGS) Solar Array”, Journal of Spacecraft and Rockets, Vol. 42, No. 3, 2005, pp. 457-466

${ }^{6}$ Dec, J.A., Amundsen, R. M., “A Thermal Analysis Approach for the Mars Odyssey Spacecraft's Solar Array”, Proposed paper for the AIAA 36 ${ }^{\text {th }}$ Thermophysics Conference, Orlando, Florida, June 23-26, 2003.

${ }^{7}$ Dec, John A., Gassbarre, Joseph F, and Amundsen, Ruth M., "Thermal Modeling of the Mars Reconnaissance Orbiter's Solar Array and Instruments During Aerobraking", to be presented at the International Conference On Environmental Systems,

${ }^{8}$ Dec, John A., Gasbarre, Joesph, and George, Benjamin, E. LT., "Thermal Analysis and Correlation of the Mars Odyssey Spacecrafts' Solar Array During Aerobraking Operations", AIAA 2002 Astrodynamics Conference, Monterey, California, AIAA 2002-4536, August 2002.

${ }^{9}$ Wright, M.J., Bose, D., Chen,Y.-K.,’Probabilistic Modeling of Aerothermal and Thermal Protection Material Responose Uncertainties", to appear in AIAA Journal. 